\title{
IMPLEMENTATION AND VALIDATION OF A BREAKER MODEL IN A FULLY NONLINEAR WAVE PROPAGATION MODEL
}

\author{
Stéphan N. Guignard ${ }^{1}$ and Stéphan T. Grilli ${ }^{1}$, M.ASCE
}

\begin{abstract}
A spilling breaker model is implemented in a two-dimensional fully nonlinear coastal wave propagation model. A maximum surface slope breaking criterion is used to identify breaking waves within the incident wave train. Energy dissipation is achieved by specifying an absorbing surface pressure over breaking wave crest areas. The pressure is proportional to the normal particle velocity on the free surface. The instantaneous power dissipated in each breaking wave is specified proportional to the dissipation in a hydraulic jump of identical characteristics. Computations for a periodic wave shoaling and breaking over a plane slope are compared to laboratory experiments. The agreement is quite good, although more work remains to be done in refining the breaker model parameters.
\end{abstract}

\section{INTRODUCTION}

Coastal wave propagation models have been developed, in recent years, following three main approaches : (i) the solution of Fully Nonlinear Potential Flow (FNPF) equations, typically, in an Eulerian-Lagrangian formulation based on a Boundary Integral Equation representation of the solution (e.g., Grilli et al. 1989; Ohyama and Nadaoka 1991); (ii) the derivation and (usually finite difference) solution of approximate long wave equations (such as Boussinesq equations with improved dispersion and/or nonlinear characteristics, e.g., Schäffer et al. 1993; Wei et al. 1995); (iii) the solution of Euler/Navier-Stokes equations, using a Volume of Fluid (VOF) or a Mark And Cell (MAC) method (e.g., Lin and Liu 1998; Guignard et al. 1999).

Models based on approach (i) have proved very accurate for modeling highly nonlinear waves shoaling over arbitrary bottom topography in two-dimensions (2D) (e.g., Grilli et al. 1994; Ohyama et al. 1994; Grilli and Horrillo 1999). In these models, the overturning of one wave can be accurately modeled, up to impact of the breaker jet on the free surface (e.g., Grilli et al. 1997; Li and

\footnotetext{
${ }^{1}$ Dept. of Ocean Engng., University of Rhode Island, Narragansett, RI 02882, USA. E-mail: grilli@oce.uri.edu.
} 

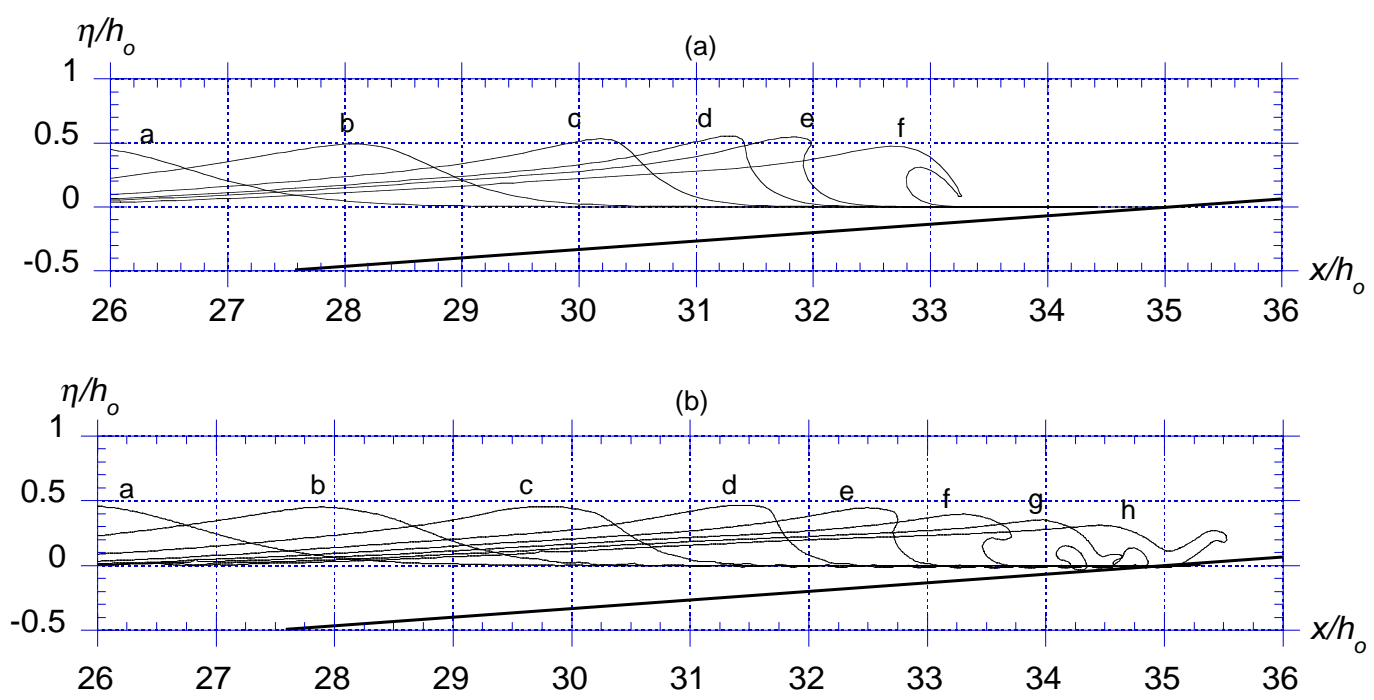

FIG. 1. (a) FNPF and (b) coupled VOF/FNPF computation of the breaking of a solitary wave on a 1:15 slope (based on Guignard et al. 1999).

Raichlen 1998; Grilli et al. 1998; Fig. 1a). [Note that similar FNPF computations of three-dimensional overturning nearshore waves have also recently been performed by Grilli et al. 2001.] Computations, however, break down beyond jet impact, unless waves are "numerically" prevented from breaking. Since potential flow models do not normally include energy dissipation terms (e.g., representing bottom friction and wave breaking effects), wave breaking is typically prevented by dissipating incident wave energy in so-called Absorbing Beaches (AB), using a surface pressure and/or actively absorbing boundaries (e.g., Clément 1996; Grilli and Horrillo 1997). This is further detailed below.

FNPF equations are only approximately solved in models based on approach (ii), in a depth integrated formulation (precluding the modeling of steep bottom obstacles), in which wave nonlinearity and dispersion are only represented to a certain order. These models, however, are less computationally demanding than in approach (i), which makes it possible to perform computations over horizontal 2D domains of meaningful size. Also, energy dissipation terms can be directly introduced in the equations (e.g., Karambas and Koutitas 1992; Schäffer et al. 1993; Skotner and Apelt 1999; Kennedy et al. 2000).

In approach (iii), full dynamic equations are solved, either for the mean fields, on a coarser global grid, together with a turbulence model representing dissipation at sub-grid scales (Lin and Liu 1998), or directly on a finer grid (Guignard et al. 2001). In the latter case, very detailed shape and kinematics of breaking waves can be obtained. However, computational cost rapidly becomes prohibitive, even for small size vertical 2D domain. In that respect, a more efficient solution, proposed by Guignard et al. (1999), has been to combine approaches (i) and (iii), i.e., to couple a FNPF model for representing shoaling waves, to a VOF model for representing breaking waves (Fig. 1b). 
In the present work, we use the 2D-FNPF model, initially developed by Grilli et al. (1989) (GSS), to study coastal wave shoaling and breaking over slopes. Grilli and Subramanya (1996) implemented more accurate discretization methods and a node regridding technique in this model, and were able to accurately calculate solitary waves breaking over mild and steep slopes (Grilli et al. 1997). In particular, they were able to predict whether and how (i.e., spilling, plunging, or surging) waves break on a plane slope. Grilli and Horrillo (1997) (GH) implemented exact periodic wave generation (streamfunction wave solution), and numerical energy absorption in the model. Absorption of energy was achieved through a combination of a surface pressure, working against waves, and an open active absorbing boundary, within an AB. They were able to calculate numerically-exact fully nonlinear properties of periodic waves shoaling over mild monotonous slopes, such as wave height and celerity variations (Grilli and Horrillo 1996), and wave transformations over barred-beaches (Grilli and Horrillo 1999). Various comparisons of these numerical results with laboratory experiments showed a very good agreement.

In a FNPF model, periodic waves, shoaling over a sloping bottom, can thus be effectively absorbed in an AB, before they start overturning (Grilli and Horrillo 1997; AB in Fig. 2). For non-periodic incident waves and/or irregular bathymetry, however, it is desirable to have a means of both preventing waves from overturning during shoaling, while gradually dissipating the energy of such individual breaking waves, in relation with the physical rate of energy dissipation in actual waves. This approach is followed in some Boussinesq models, in which empirical eddy viscosity terms are added in the momentum equation, combined to a breaking criterion, such as a maximum angle on the wave front face (Schäffer et al., 1993). The eddy viscosity is calibrated based on laboratory experiments. Here, a spilling breaker model is implemented in the NWT (from the breaking point $x=x_{b}$ to $x_{a}$ in Fig. 2), in which the instantaneous rate of energy dissipation for each broken wave is assumed to be that of a hydraulic jump. This analogy was suggested by Svendsen et al. (1978), based on experiments, to estimate the rate of energy dissipation in surf-zone waves.

A maximum/minimum front slope criterion, similar to Schäffer et al.'s, is used to determine whether a wave starts or stops breaking. Following the method used in GH's AB, an absorbing surface pressure distribution is specified over each breaking wave crest area, from the point where normal velocity changes sign behind the crest, to the similar point on the wave front face. The (negative) work produced by this pressure against the wave is calibrated in real time to be proportional to the energy dissipated in an inverted hydraulic jump. This requires knowing values of instantaneous wave characteristics such as wave height $H$, celerity $c$, and depth below crest $h_{c}=h+H$, and trough, $h_{t}$ (Fig. 3). Hence, a wave tracking algorithm is developed, in which individual waves are identified and followed throughout their shoaling and breaking in the model, while the breaking creterion is being checked. The spilling breaker model is calibrated by comparing results to laboratory experiments for mean wave height, mean-water- 


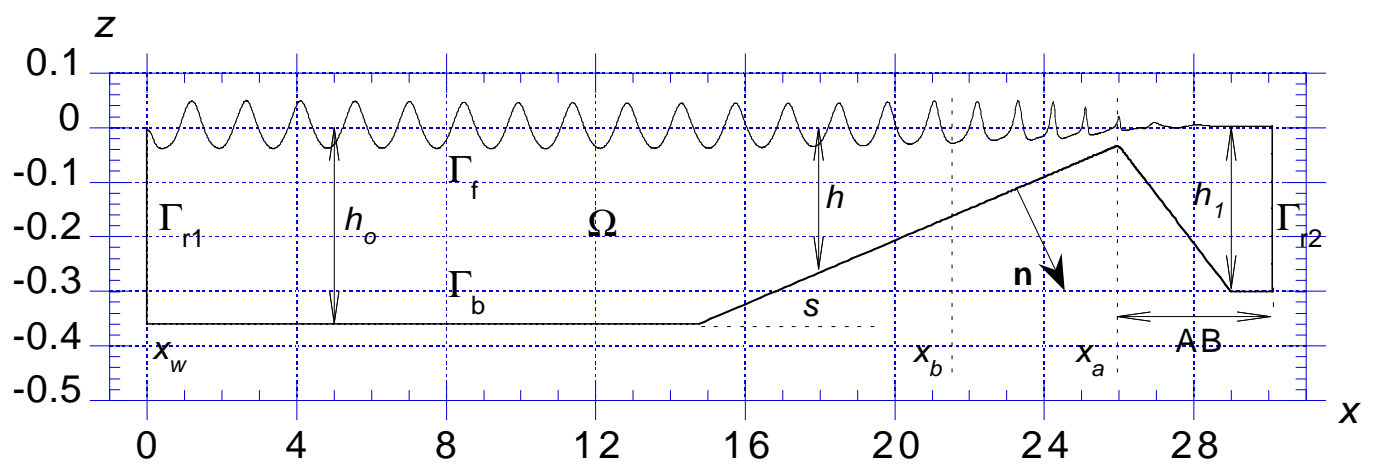

FIG. 2. Sketch of FNPF model for periodic wave shoaling and breaking computations.

level (MWL), and wave celerity variations, during shoaling of periodic waves generated by a piston wavemaker (Hansen and Svendsen 1979).

Note that, in recent years, other criteria have been proposed to detect and/or remove breaking in wave models. Thus, Subramani et al. (1998) developed a maximum surface curvature criterion, to identify deep water breaking waves ( $\kappa H \leq 0.7$, with $\kappa$ the crest curvature), and Gentaz and Alessandrini (2000) used a criterion based on a threshold vertical pressure gradient at the free surface $\left(\frac{\partial p}{\partial z} \geq \rho g\right)$. Finally, note the method introduced by Wang et al. (1995) to suppress breaking in their computations, in which water is simply peeled away from wave crests reaching a limiting height (this, however, violates mass conservation).

\section{THE NUMERICAL WAVETANK}

\section{Governing equations and boundary conditions}

Equations for the 2D-FNPF model can be found in the references (GSS,GH). The velocity potential $\phi(x, z, t)$ is used to describe inviscid irrotational flows in the vertical plane and the velocity is defined as $\boldsymbol{u}=\boldsymbol{\nabla} \phi$ (Fig. 2). Continuity equation in the fluid domain $\Omega(t)$ with boundary $\Gamma(t)$ is a Laplace's equation for the potential. On the free surface $\Gamma_{f}(t), \phi$ satisfies fully nonlinear kinematic and dynamic boundary conditions. Along the stationary parts of the boundary such as bottom $\Gamma_{b}$ and $\Gamma_{r 2}$, a no-flow condition is prescribed. For comparing results with experiments, periodic waves are generated on boundary $\Gamma_{r 1}(t)$ using a solid piston wavemaker $\left(x=x_{w}\right)$, moving according to a first-order cnoidal wave solution.

\section{Numerical model}

At each time $t$, the model solves a Boundary Integral Equation (BIE), representing continuity equation, using a higher-order Boundary Element Method (BEM) (see GSS, and Grilli and Subramanya 1996, for details). Free surface boundary conditions are time integrated based on two second-order Taylor series expansions expressed in terms of a time step $\Delta t$ and the Lagrangian time derivative of free surface position and potential. Detailed expressions for the Taylor 
series are given in GSS. The optimal time step for each time is selected based on a mesh Courant number $\mathcal{C}_{o}(t) \simeq 0.45$.

In computations involving finite amplitude waves, mean drift currents occur which continuously move discretization nodes/Lagrangian markers forward in the model. To counteract this effect, Grilli and Subramanya (1996) developed regridding methods in which nodes can be redistributed at constant arclength intervals over specified regions of the free surface. Here, to limit the number of nodes on the free surface and the computational cost, the initial horizontal node spacing $\Delta x_{0}$ is gradually reduced over the slope, to match the reduction in wavelength due to shoaling and maintain a node density of at least 15 nodes per waves (from an initial 20 nodes per wavelength over the constant depth part of the NWT). Hence, the method of constant arclength regridding can not be applied. Instead, a new regridding method was used in which the initial ratio of each BEM element length to the total length of the free surface is maintained for all times.

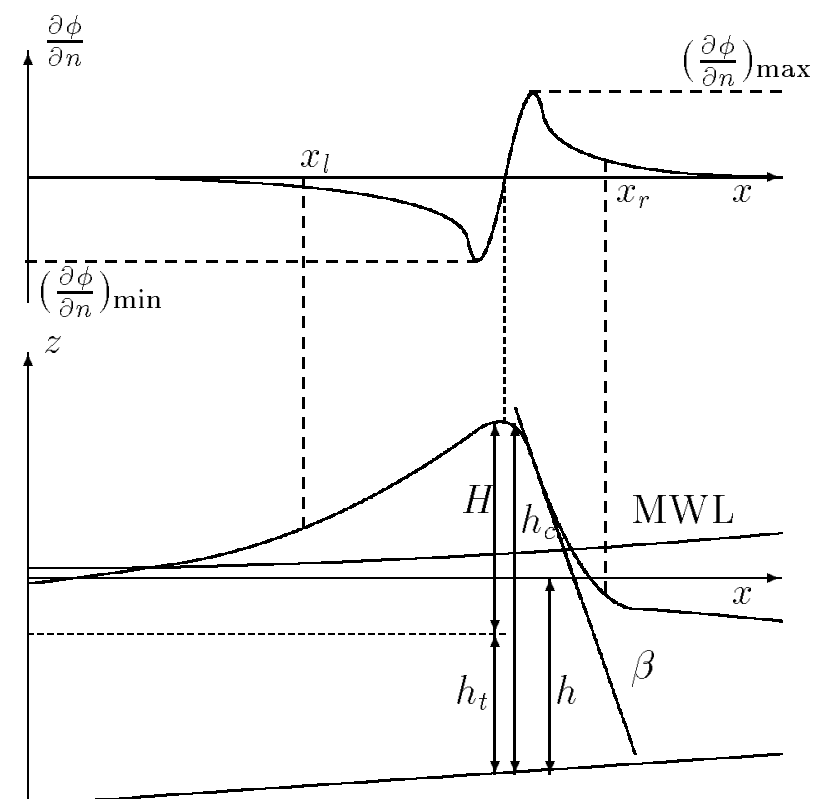

\section{FIG. 3. Definition of geometric parameters for the breaker model. [Note, $H$ is defined as the height between a crest and the previous trough.]}

\section{Wave energy absorption}

The same method as in GH's AB is used to selectively absorb energy from waves, identified to break for $x \leq x_{a}$, i.e., an absorbing surface pressure is specified in the dynamic free surface condition (with $z=\eta$ ), proportional to the normal particle velocity on the free surface. An AB is still used to absorb residual wave energy exiting at the top of the slope, for $x>x_{a}$ (Fig. 2). [To create additional wave reduction through de-shoaling, the bottom geometry within the $\mathrm{AB}$ is specified somewhat similar to a natural bar, with a depth increasing to $h=h_{1}$.] A wave tracking algorithm (detailed below) identifies breaking waves, 
within the incident wave train, based on a breaking criterion. The breaker model is assumed to extend from the crest of each such wave to two points on each side of the crest, where $\left|\frac{\partial \phi}{\partial n} /\left(\frac{\partial \phi}{\partial n}\right)_{\min , \max }\right|>\varepsilon\left(x_{l} \leq x \leq x_{r}\right)$, with $\varepsilon$ is a small threshold value (Fig. 3). [|( $\left.\frac{\partial \phi}{\partial n}\right)_{\min , \max } \mid$ are defined as the maximum absolute normal velocity for each side of the wave.] Over each breaker, the absorbing pressure is defined as,

$$
p_{b m}(x, \eta, t)=\nu_{b m}(x) \frac{\partial \phi}{\partial n}(\eta(x, t))
$$

in which $\nu_{b m}=\nu_{b o} S(x)$, with $S(x)$ a breaker shape function providing a smooth transition from areas without the absorbing pressure, to the breaker regions over each breaking wave.

The instantaneous power dissipated by each breaking wave is given by,

$$
\mathcal{P}_{b}=\int_{x_{l}}^{x_{r}} p_{b} \frac{\partial \phi}{\partial n} \mathrm{~d} \Gamma=\nu_{b o} \int_{x_{l}}^{x_{r}} S(x)\left(\frac{\partial \phi}{\partial n}\right)^{2} \mathrm{~d} \Gamma
$$

and is assumed to be proportional to the power dissipated in a turbulent hydraulic jump (e.g., Lamb 1932, p 280),

$$
\mathcal{P}_{h}=\rho g c \frac{h H^{3}}{4 h_{c} h_{t}}
$$

where $H$ denotes the wave height, $h_{t}$ the water depth below trough, $h_{c}=h_{t}+H$ the water depth below crest, and (here) $c$ is the absolute wave crest phase speed. Following Svendsen et al. (1978), we define, $\mathcal{P}_{b m}=\mu \mathcal{P}_{h}$, with $\mu \simeq 1.5$. All calculations done, the instantaneous value of the breaker coefficient $\nu_{b o}$ for each breaking wave is found based on the values of both wave and breaker parameters $\left(H, h, h_{t}, \mu, \varepsilon\right)$, and the wave shape and kinematics in between $x_{l}$ and $x_{r}$.

\section{Wave tracking algorithm and breaking criteria}

For each time $t,(x, z)$ locations of local maxima and minima in surface elevation $\eta(x, t)$ are identified. The crest of each wave is calculated as the highest elevation in between two successive minima. A wave is defined only if its height $H$ is greater than a specified fraction of the incident wave height $H_{0}$ (typically one-tenth). This avoids including secondary wave crests created during shoaling.

For each wave defined this way, the algorithm finds which wave $i=1, \ldots$ it corresponded to at the previous time $t-\Delta t$. The search for the right wave is accelerated by extrapolating the crest position of each earlier wave $i$, to time $t$,

$$
\tilde{x}_{c}^{i}(t) \simeq x_{c}^{i}(t-\Delta t)+c^{i} \Delta t
$$

where, $c^{i} \simeq \frac{\partial \phi}{\partial t}\left(x_{c}^{i}\right) / \frac{\partial \phi}{\partial x}\left(x_{c}^{i}\right)$ is the $i$ s wave crest celerity at time $t-\Delta t$ (assuming a permanent form for the wave over time $\Delta t$ ), and comparing it to the crest position $x_{c}(t)$ found for the wave currently under consideration. Wave heights $H^{i}(x, t)$ are stored for each wave after final identification is made as well as other geometric parameters needed to calculate the hydraulic jump power dissipation 

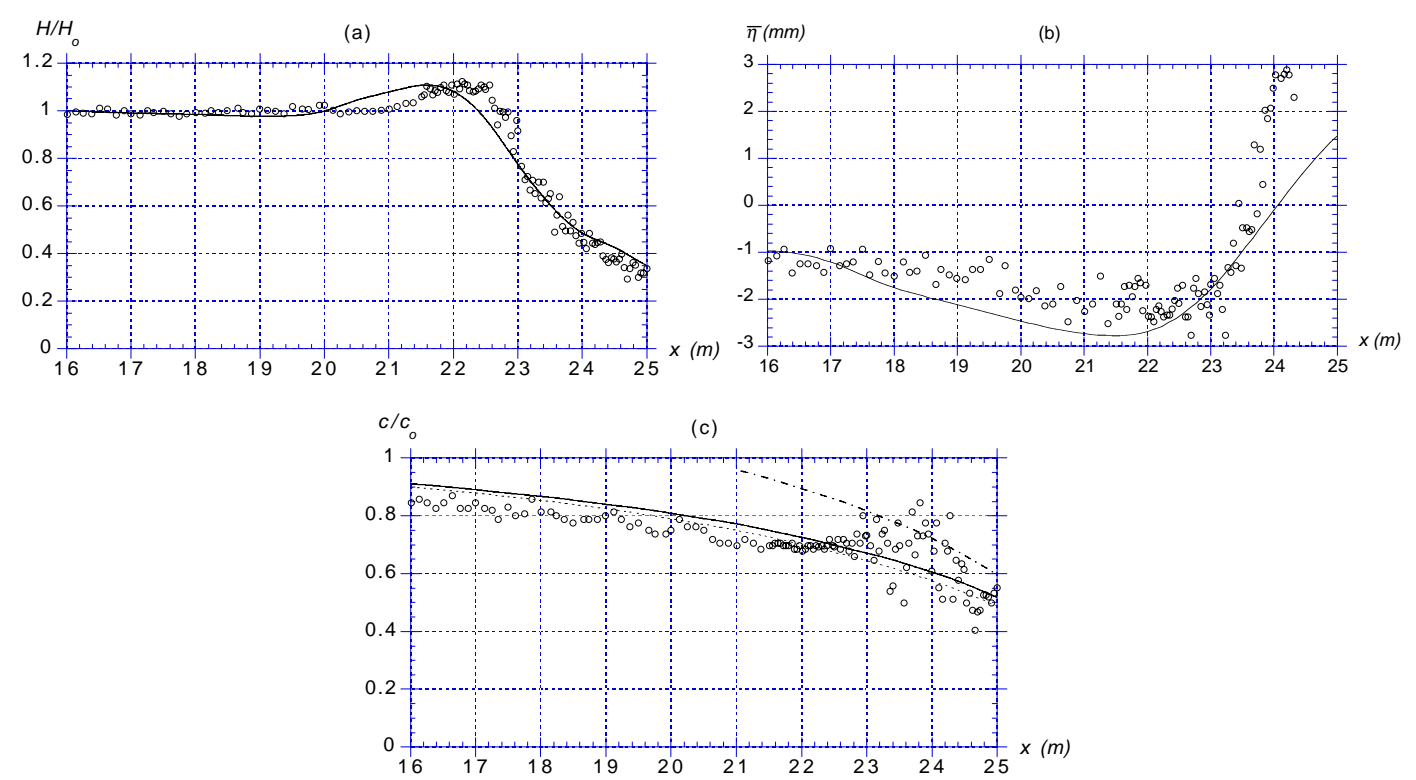

FIG. 4. Calculated (--) : (a) mean wave height; (b) MWL; and (c) mean celerity. (o) are experimental data. In (c) : NSW (- - ) and LWT (- - ).

using Eq. (3). Crest trajectories $x_{c}^{i}(t)$ are calculated for each wave $i=1, \ldots$ in the incident wave train, and celerities are calculated as the time derivatives of these. Wavelengths are readily found as $L^{i}=c^{i} T$, assuming a constant wave period.

The breaking criterion (a simple wave front angle threshold criteria, $\beta>\beta_{\max }$ ) is checked for each wave $i$ identified at time $t$, to decide whether it breaks or not. For those waves $j$ that break, the procedure described in the previous section is applied to calculate the interval $x_{l}^{j} \leq x \leq x_{r}^{j}$, in between which an absorbing pressure $p_{b m}^{j}$ should be applied (Fig. 3), according to Eq. (1).

\section{APPLICATIONS}

One computation is presented here, representing laboratory experiments by (Hansen and Svendsen 1979), for periodic waves with : $H_{0}=0.095 \mathrm{~m}$ (at the toe of the slope), $T=1 \mathrm{~s}$, and $h_{\circ}=0.36 \mathrm{~m}$, shoaling over a $s=1 / 34.26$ plane slope for $x \geq 14.78 \mathrm{~m}$ (Fig. 2). In the experiments, waves were generated by a piston wavemaker at $x=0$, using a second-order wave generation method. Variations of wave-averaged, wave height $H$, mean-water-level (MWL) $\bar{\eta}$, and celerity $c$, were measured as a function of $x$. The same tank geometry and wave characteristics are used in the model. Wave generation is performed using a piston wavemaker moved according to a first-order cnoidal wave solution, with wave height $0.095 \mathrm{~m}$. It was observed that generated waves slightly adjusted their shape and height as they propagated over constant depth down the modeled tank, likely due to nonlinear effects. The incident wave finally reached a stabilized height $H_{0}=0.085 \mathrm{~m}$ at the toe of the slope i.e., a smaller value than in the corresponding experiments (which used a wave height $0.1 \mathrm{~m}$ at the wavemaker). This initial comparison of 
numerical results with experiments was carried out using those slightly smaller generated waves, after scaling by the wave height.

An AB, with water depth deepening to $h_{1}=0.3 \mathrm{~m}$, is specified at the top of the slope for $x \geq x_{a}=25.96 \mathrm{~m}$ and $x \leq 30$, with $\nu_{a \circ}=0.01$. The minimum depth on the slope at $x=x_{a}$ is thus $h_{a}=0.034 \mathrm{~m}$. Breaking is assumed to occur when the maximum wave front angle reaches $\beta_{\max }=37^{\circ}$, with $\varepsilon=10^{-5}$. Finally, we select $\mu=1.5$.

BEM discretization and time step parameters are selected as described before. There is a total of $N=672$ nodes on the boundary and the initial time step is $\Delta t_{0}=0.01 \mathrm{~s}$. A total of 5000 time steps were run in these computations. The maximum relative numerical error, with respect to the initial volume of the tank $\left(V_{o}=8.322 \mathrm{~m}^{3} / \mathrm{m}\right)$ was only $0.011 \%$ after 3500 iterations (time $t=21 \mathrm{~s}$ ). At this stage, the model reached an almost steady state in which larger waves kept entering the AB from the top of the slope. This led to somewhat larger numerical errors. After 5000 iterations, at time $t=30.04 \mathrm{~s}$ the volume error increased to $0.087 \%$, which is still quite small.

Fig. 4 show results of computations averaged over 6 successive waves, after computations reach an almost steady state. Breaking occurs in average for $x_{b} \simeq 21.5 \mathrm{~m}$ (Fig. 4a). There is a shoaling region for $x<x_{b}$, where wave height $H(x)$ eventually slightly increases, and a surf-zone region beyond breaking, where $H$ decreases. The agreement with experiments is quite good although breaking occurs slightly too early (i.e. for too small a $x$ ) in the model. The agreement of computed MWL with experiments is also good for $x<24 \mathrm{~m}$ (Fig. 4b). Fig. 4c shows the calculated celerity, together with the celerity predicted by linear wave theory, $c_{\mathrm{LWT}}=c_{o} \tanh k h$ (with $c_{o}=g T / 2 \pi=1.56 \mathrm{~m} / \mathrm{s}$, wavenumber $k=2 \pi / L$, and wavelength $L=c T$ ), and that of the Nonlinear Shallow Water equations,

$c_{\mathrm{NSW}}=\sqrt{g(H+h)}$ (using the FNPF results for $H$ ). The agreement of $c$ and $c_{\text {LWT }}$ with experiments is quite good during the shoaling part $(x<21.5 \mathrm{~m})$, considering the difficulty in accurately measuring celerities reported by Hansen and Svendsen, and the small difference in $H_{\circ}$ value. Beyond breaking, experimental results show a large variance, maybe due to difficulties in identifying crests in the experiments. Experimental results however quite well illustrate the effects of amplitude dispersion, which lead to a larger celerity for larger waves. This is also seen in larger FNPF results as compared to linear wave theory. [The marked increase in celerity seen beyond breaking in experiments, and also predicted in FNPF computations reported by Wei et al. 1995, is not modeled here since the formation of a fast forward moving jet is prevented.] Nonlinear shallow water equations overpredict wave celerity in most places.

\section{CONCLUSIONS}

Results show, the spilling breaker model implemented in the FNPF model correctly accounts for the overall physics of periodic waves breaking over a sloping bottom, i.e., the approximate location and height of breaking, and the rate of energy dissipation in breakers, leading to a reduction in wave height and an increase 
in MWL in the surf-zone. The good agreement of numerical and experimental results confirms the relevance of the hydraulic jump analogy, with $\mu=1.5$. More work is required to see whether this $\mu$ value is general.

The $\beta_{\max }$ value in the breaking criterion is larger than typically used in Boussinesq models, likely because the FNPF model produces steeper waves and thus delays the onset of breaking. Since breaking still occurs too soon, it would be of interest to try and increase $\beta_{\max }$ further. A limit, however, is put on this increase in the sense that, for too large a $\beta_{\max }$ value, it may be difficult to absorb enough (or quickly enough) of the wave energy to prevent wave overturning from occurring in the model.

\section{ACKNOWLEDGMENT}

This research was supported by the Office of Naval Research, under grant N00014-99-1-0439 from the US Department of the Navy, Office of the Chief of Naval Research. The information reported in this work does not necessarily reflect the position of the US Government.

\section{REFERENCES}

Clément, A. (1996). "Coupling of two absorbing boundary conditions for $2 \mathrm{~d}$ timedomain simulations of free surface gravity waves." J. Comp. Phys., 26, 139-151.

Gentaz, L. and Alessandrini, B. (2000). "Detection of wave breaking in a $2 \mathrm{~d}$ viscous numerical wave tank." Proc. 10th Offshore and Polar Engng. Conf., Vol. 3. 263-270.

Grilli, S., Guyenne, P., and Dias, F. (2001). "A fully nonlinear model for threedimensional overturning waves over arbitrary bottom." Intl. J. Numer. Methods in Fluids, 35(7), 829-867.

Grilli, S. and Horrillo, J. (1996). "Fully nonlinear properties of periodic waves shoaling over mild slope." Proc. 25th Intl. Conf. on Coastal Engng., Vol. 1. ASCE Edition, 717-730.

Grilli, S. and Horrillo, J. (1997). "Numerical generation and absorption of fully nonlinear periodic waves." J. Engng. Mech., 123(10), 1060-1069.

Grilli, S. and Horrillo, J. (1999). "Shoaling of periodic waves over barred-beaches in a fully nonlinear numerical wave tank." Intl. J. Offshore and Polar Engng., 9(4), 257-263.

Grilli, S., Skourup, J., and Svendsen, I. (1989). "An efficient boundary element method for nonlinear water waves." Engng. Analysis with Boundary Elements, $6(2), 97-107$.

Grilli, S. and Subramanya, R. (1996). "Numerical modeling of wave breaking induced by fixed or moving boundaries." Computational Mech., 17, 374-391.

Grilli, S., Subramanya, R., Svendsen, I., and Veeramony, J. (1994). "Shoaling of solitary waves on plane beaches." J. Waterway Port Coastal and Ocean Engng., $120(6), 609-628$.

Grilli, S., Svendsen, I., and Subramanya, R. (1997). "Breaking criterion and characteristics for solitary waves on slopes." J. Waterway Port Coastal and Ocean Engng., 123(3), 102-112. 
Grilli, S., Svendsen, I., and Subramanya, R. (1998). "Breaking criterion and characteristics for solitary waves on slopes - closure." J. Waterway Port Coastal and Ocean Engng., 124(6), 333-335.

Guignard, S., Grilli, S., Marcer, R., and Rey, V. (1999). "Computation of shoaling and breaking waves in nearshore areas by the coupling of BEM and VOF methods." Proc. 9th Offshore and Polar Engng. Conf., Vol. 3. 304-309.

Guignard, S., Marcer, R., Rey, V., Kharif, C., and Fraunié, P. (2001). "Solitary wave breaking on sloping beaches : $2 \mathrm{D}$ two-phase flow numerical simulation by sl-vof method." Eur. J. Mech. B - FLuids, 20, 57-74.

Hansen, J. and Svendsen, I. (1979). "Regular waves in shoaling water. Experimental data." Series paper No. 21, Technical University of Denmark, Institute of Hydrodynamics and Hydraulic Engng.

Karambas, T. and Koutitas, C. (1992). "A breaking wave propagation model based on the Boussinesq equations." Coastal Engng., 18, 1-19.

Kennedy, A., Chen, Q., Kirby, J., and Dalrymple, R. (2000). "Boussinesq modeling of wave transformation, breaking, and runup. I: 1D." J. Waterway Port Coastal and Ocean Engng., 126(1), 39-47.

Lamb, H. (1932). Hydrodynamics. Dover Publications, New York, 6th edition.

Li, Y. and Raichlen, F. (1998). "Breaking criterion and characteristics for solitary waves on slopes - discussion." J. Waterways Port Coastal Ocean Engng., 124(6), 329-333.

Lin, P. and Liu, P. (1998). "A numerical study of breaking waves in the surf zone." J. Fluid Mech., 359, 239-264.

Ohyama, T. and Nadaoka, K. (1991). "Development of a numerical wave tank for analysis of nonlinear and irregular wave fields." Fluid Dyn. Res., 8, 231-251.

Ohyama, T., S., B., Nadaoka, K., and Battjes, J. (1994). "Experimental verification of numerical model for nonlinear wave evolution." J. Waterway Port Coastal and Ocean Engng., 120(6), 637-644.

Schäffer, H., Madsen, P., and Deigaard, R. (1993). "A Boussinesq model for wave breaking in shallow water." Coastal Engng., 20, 185-202.

Skotner, C. and Apelt, C. (1999). "Application of a Boussinesq model for the computation of breaking waves. Part 1 : Development and verification." Ocean Engng., 26, 907-926.

Subramani, A., Beck, R., and Schultz, W. (1998). "Suppression of wave breaking in nonlinear water wave computations." Proc. 13th Intl. Workshop Water Waves and Floating Bodies. Delft University of Technology, 139-141.

Svendsen, I., Madsen, P., and Hansen, J. (1978). "Wave characteristics in the surf zone." Proc. 16th Intl. Coastal Engng. Conf. ASCE Edition, 521-539.

Wang, P., Yao, Y., and Tulin, M. (1995). "An efficient numerical tank for nonlinear water waves, based on the multi-subdomain approach with BEM." Intl. J. Num. Methods in Fluids, 20, 1315-1336.

Wei, J., Kirby, J., and Grilli, S. (1995). "A fully nonlinear Boussinesq model for surface waves. Part 1. Highly nonlinear unsteady waves." J. Fluid Mech., 294, 71-92. 